\title{
Phase Transitions for Two-Dimensional Models with Isotropic Short-Range Interactions and Continuous Symmetries
}

\author{
S. B. Shlosman \\ Institute of the Information-Transmission Problems of the Academy of Sciences of the USSR, Moscow, \\ USSR
}

\begin{abstract}
Using the powerful method of reflection-positivity and chess-board estimates, we prove the existence of phase transition for certain class of isotropic short-range interactions with continuous symmetry, provided that the dimension of the lattice is at least two, and the temperature is low enough.
\end{abstract}

\section{Introduction}

There has been great progress recently in proving the existence of phase transitions for systems of statistical mechanics. The powerful methods of reflection-positivity and chess-board estimates were applied to both classical and quantum cases [1]. This made it possible to handle many anisotropic nearest-neighbor interactions [1] and to recover some results of Dyson and Kunz-Pfister for long-range isotropic interactions [2]. Further results of the authors of [2] are announced for Coulomb gases and quantum field theory.

The purpose of this paper is to show the possibility of applying the methods of $[1,2]$ to isotropic short-range interactions with continuous symmetry. For such interactions there exists a general result of [3] about absence of breakdown of continuous symmetry. So the one-point correlation functions must be the same in all phases. Indeed, in models we consider, the difference between phases is manifested by the difference between two-point correlation functions of these phases. The phases we will construct in Sect. 2 are translation invariant (after suitable change of coordinates). It does not contradict, however, results of $[4,5]$ about uniqueness of translation-invariant states for some $S^{1}$-invariant interactions, because we are dealing with an entirely different class of models. Nevertheless, several correlation inequalities of $[4,5]$ are also valid for our interactions (those of Ginibre type).

Finally, about the content of the paper. Section 2 contains the proof of the simplest variant of our results. This is done for the sake of clarity. The general result is formulated in Sect. 3. As the proof of it is essentially the same, it is omitted. The paragraph is concluded by some discussions. 


\section{The Main Result}

Let $\mathbb{Z}^{2}$ be two-dimensional lattice, $L_{e} \subset \mathbb{Z}^{2}$ be any sublattice of index two, and $L_{0}=\mathbb{Z}^{2} \backslash L_{e}$ be the coset. We'll consider the model with the hamiltonian

$$
\begin{aligned}
-H= & J_{1} \sum_{i, j \in L_{e},|i-j|=\sqrt{2}} \cos \left(\varphi_{i}-\varphi_{j}\right) \\
& +J_{2} \sum_{i, j \in L_{o},|i-j|=\sqrt{2}} \cos \left(\varphi_{i}-\varphi_{j}\right) \\
& +J_{3} \sum_{i \in L_{e}, j \in L_{o},|i-j|=1} \cos \left[2\left(\varphi_{i}-\varphi_{j}\right)+\pi\right],
\end{aligned}
$$

where || means euclidean distance, $\varphi_{i} \in S^{1}, J_{1}, J_{2}, J_{3} \neq 0$. Without loss of generality we can suppose $J_{1}, J_{2}, J_{3}>0$. The free measure is simply the Lebesque measure $d \varphi, \int d \varphi=2 \pi$.

Now, the first and the second terms in the hamiltonian are the interaction between spins on $L_{e}$, and $L_{o}$. This interaction is attractive, and via it the spins on each sublattice have a tendency of being parallel. The third term represents the interaction between sublattices, and it is of such a tipe, that the spins have a tendency to be perpendicular. The hamiltonian $H$ is evidently rotation-invariant. Moreover, it is also invariant under the transformation

$$
\varphi_{i} \rightarrow \varphi_{i}, \quad \varphi_{j} \rightarrow \varphi_{j}+\pi ; \quad i \in L_{e}, \quad j \in L_{o} .
$$

Thus, the groundstates of $H$ are of the form:

$$
\varphi_{i} \equiv \alpha, \quad i \in L_{e}, \quad \varphi_{j} \equiv \beta, \quad j \in L_{o}
$$

and

$$
|\alpha-\beta|=\frac{\pi}{2} \bmod \pi
$$

Being applied to this model, our main result implies the following:

Theorem 1. There exist at least two different phases for the interaction (1), provided the temperature is low enough.

Both of them are, of course, rotation-invariant [3], but with probability tending to 1 as temperature goes to zero, the rotation from $\varphi_{(0,0)}$ to $\varphi_{(0,1)}$ is clockwise in one face, and counterclockwise in another (in a typical configuration).

The proof of this theorem is based on the reflection-positivity and chess-board estimates method. As for reflection-positivity of the hamiltonian $H$ according to reflections in the coordinate lines of $\mathbb{Z}^{2}$ and their $\mathbb{Z}^{2}$-translates, it can be seen exactly in the same way, as it was done in [1] for the case of anharmonic crystal. Indeed, after fixing any configuration on such a line (=one-dimensional sublattice for $\mathbb{Z}^{2}$, = union of two circles for two-dimensional torus), two resulting families of spins are conditionally independent and identically distributed.

In order to introduce contours, we will slightly rewrite the hamiltonian $H$. To do this we will use the trick previously used by Pirogov [6], when discussing the anharmonic crystal result of $[1]$. Let $I_{1}=2 J_{1}, I_{2}=2 J_{2}, I_{3}=J_{3} / 2$, and let $\left\{c_{s}\right\}$ be 
the collection of unit closed squares of $\mathbb{Z}^{2}$, where $s$ runs over the sites of dual lattice. Then

$$
\begin{aligned}
H= & \sum_{s} H_{c_{s}}, \\
-H_{c_{s}}= & I_{1} \cos \left(\varphi_{i}-\varphi_{j}\right)+I_{2} \cos \left(\varphi_{k}-\varphi_{l}\right) \\
& +I_{3}\left\{\cos \left[2\left(\varphi_{i}-\varphi_{k}\right)+\pi\right]+\cos \left[2\left(\varphi_{i}-\varphi_{l}\right)+\pi\right]\right. \\
& +\cos \left[2\left(\varphi_{j}-\varphi_{k}\right)+\pi\right]+\cos \left[2\left(\varphi_{j}-\varphi_{l}\right)+\pi\right],
\end{aligned}
$$

where $\{i, j\}=c_{s} \cap L_{e},\{k, l\}=c_{s} \cap L_{o}$. Now let a configuration $\left\{\varphi_{t}, t \in \mathbb{Z}^{2}\right\}$ be given. We call a square $c_{s}$ regular, if

$$
\begin{aligned}
\left|\varphi_{i}-\varphi_{j}\right| & <\delta, \quad\left|\varphi_{k}-\varphi_{l}\right|<\delta \\
\left|\frac{\pi}{2}-\right| \varphi_{\alpha}-\varphi_{\beta}|| & <\delta \bmod \pi \text { for all } \alpha \in\{i, j\}, \quad \beta \in\{k, l\} .
\end{aligned}
$$

Here $i, j, k, l \in c_{s},|i-j|=|k-l|=\sqrt{2}$ and $\delta$ is some positive real, which we will specify later. Finally, we call a square $c_{s}$ irregular, if it is not regular.

A sequence $c_{s(k)}$ is called a path, if for any $k$ two squares $c_{s(k)}$ and $c_{s(k+1)}$ have a common edge. If $\delta$ is less than, say, $\pi / 10$, then we have two tipes of the regular squares, according to the direction of the rotation from $L_{e}$-spins to $L_{o}$-spins. We'll call a regular square to be of $(+)$-type, if the rotation from $L_{e}$-spins to $L_{o}$-spins is clockwise, and of (-)-type, if it is counterclockwise. It is easy to see, that any path $c_{s(k)}$, which connects regular squares of different types, also contains at least one irregular square between them. So the following definition agrees with the usual feeling:

For a given configuration $\left\{\varphi_{t}, t \in \mathbb{Z}^{2}\right\}$, we call a contour any maximal connected component of a set of irregular squares.

The reader must note, that by "square" we mean closed square, so the set of two squares, which have only one vertex in common, is connected.

Let $s, t$ be two vertices of the dual lattice, and let $P_{s}^{ \pm}$denote the characteristic function of the event that the square $c_{s}$ is of ( \pm$)$-type. To prove our theorem it is enough to show that

$$
\left\langle P_{s}^{+} P_{t}^{-}\right\rangle_{\Lambda, \beta} \rightarrow O \quad \text { as } \quad \beta \rightarrow \infty
$$

uniformly in $s, t, \Lambda$, where by \langle\rangle$_{\Lambda, \beta}$ we mean the integration respect to measure

$$
Z_{\Lambda}^{-1}(\beta) \exp \left\{-\beta H\left(\varphi_{\Lambda}\right)\right\} \prod_{i \in \Lambda} d \varphi_{i}
$$

with periodic boundary conditions,

$$
Z_{\Lambda}(\beta)=\int_{\left(S^{1}\right)^{|\Lambda|}} \exp \left\{-\beta H\left(\varphi_{\Lambda}\right)\right\} \prod_{i \in \Lambda} d \varphi_{i}
$$

and, of course, the period is supposed to be even.

To see it is enough, let us decompose any limit point $\mathscr{P}^{\beta}$ of the sequence \langle\rangle$_{\Lambda, \beta}$, $\Lambda \rightarrow \infty$ into ergodic components, $\mathscr{P}^{\beta}=\int \mathscr{P}_{\tau}^{\beta} d \tau$. Then

$$
\int P_{s}^{+} P_{t}^{-} d \mathscr{P}^{\beta}=\int\left(\int P_{s}^{+} P_{t}^{-} d \mathscr{P}_{\tau}^{\beta}\right) d \tau .
$$


If $\beta$ is large enough, then the r.h.s. of (4) is uniformly small in $s, t$. But by the ergodic theorem it tends to

$$
\int\left[\left(\int P_{s}^{+} d \mathscr{P}_{\tau}^{\beta}\right)\left(\int P_{t}^{-} d \mathscr{P}_{\tau}^{\beta}\right)\right] d \tau
$$

as $|s-t| \rightarrow \infty$. Hence there exist a set of $\tau$-s of measure almost equal to 1 , for which the product

$$
\int P_{s}^{+} d \mathscr{P}_{\tau}^{\beta} \int P_{t}^{-} d \mathscr{P}_{\tau}^{\beta}
$$

is small. But the sum of those two integrals tends to $l$ as $\beta \rightarrow \infty$ as we shall show, so one of them is near one, and the other-near zero for each $\tau$ in this set. The proof is completed by using the symmetry transformation (2).

The estimation (3) follows in the same way as in [1], using Peierls-chess-board arguments with minor modifications. There are some difference in the entropy estimate, i.e. in estimating the number of different contours with the same length. It is easy to see by induction, that for any connected graph there exists a walk on it, with the property, that one passes along every edge of the graph exactly twice. So the number of different contours consisting of $\gamma$ squares is bounded by $8^{2(\gamma-1)}$. So

$$
\left\langle P_{s}^{+} P_{t}^{-}\right\rangle_{A, \beta} \leqq \sum_{\gamma=1}^{\infty} 3 \gamma^{2} \cdot 64^{\gamma}\langle\gamma\rangle_{A, \beta},
$$

where by $\langle\gamma\rangle_{\Lambda, \beta}$ we denote the probability of finding the contour of size $\gamma$, maximalized over all possible shapes of them.

The chess-board estimate gives us the following bound for $\langle\gamma\rangle_{\Lambda, \beta}$ :

$$
\langle\gamma\rangle_{\Lambda, \beta} \leqq\left\langle P_{\Lambda}\right\rangle_{\Lambda, \beta}^{\gamma /(2|\Lambda|)}
$$

where $P_{A}$ is the "universal projector" of [1], i.e. the characteristic function of the following event: all squares $c_{s}$ are irregular. To complete the proof we need only the appropriate upper bound for $\left\langle P_{\Lambda}\right\rangle_{\Lambda, \beta}$. In other words, we must estimate the probability of every square $c_{s}$ to be irregular.

In order to this, we make first the following estimate of the statistical sum, $Z_{\Lambda}(\beta)$. (Here, $\Lambda$ is two-dimensional torus.) Let the $\operatorname{spin} \varphi_{(0,0)}$ be fixed, and consider all the configurations with $\left|\varphi_{(0,0)}-\varphi_{i}\right|<\alpha, i \in L_{e},|| \varphi_{(0,0)}-\varphi_{k}\left|-\frac{\pi}{2}\right|<\alpha, k \in L_{o}$, $\left|\varphi_{k}-\varphi_{l}\right|<\frac{\pi}{2}, k, l \in L_{o}$, where $\alpha$ is arbitrary. Then the energy of any such configuration is not more than

$$
-\beta\left(I_{1}+I_{2}+4 I_{3}\right)\left(1-8 \alpha^{2}\right)|\Lambda|,
$$

so

$$
\begin{aligned}
Z_{\Lambda}(\beta) \geqq & \exp \left\{\beta\left(I_{1}+I_{2}+4 I_{3}\right)\left(1-8 \alpha^{2}\right)|\Lambda|\right\} \\
& \cdot 2 \cdot 2 \pi \cdot(2 \alpha)^{|\Lambda|-1} .
\end{aligned}
$$

Here 2 comes from $\mathbb{Z}_{2}$-symmetry, and $(2 \pi)$ from $S^{1}$-symmetry. This bound is valid for any $\alpha$. 
Next we estimate the integral

$$
\int \exp \left\{-\beta H_{\Lambda}(\varphi)\right\} \prod d \varphi,
$$

taken over all configurations with every square being irregular. The energy of such a configuration is not less than

$$
\begin{aligned}
& -\beta\left(I_{1}+I_{2}+4 I_{3}+\frac{1}{2}\left\{I_{1}(\cos \delta-1) ; I_{2}(\cos \delta-1) ; 4 I_{3}(\cos 2 \delta-1)\right\}\right)|\Lambda| \\
& \quad \geqq-\beta\left(I_{1}+I_{2}+4 I_{3}-I \delta^{2}\right)|\Lambda|
\end{aligned}
$$

for some positive $I$ and sufficiently small $\delta$. So the integral is not more than

$$
\exp \left\{\beta\left(I_{1}+I_{2}+4 I_{3}-I \delta^{2}\right)|\Lambda|\right\}(2 \pi)^{|\Lambda|},
$$

and finally,

$$
\left\langle P_{\Lambda}\right\rangle_{\Lambda, \beta} \leqq \exp \left\{\beta|\Lambda|\left(-I \delta^{2}+8\left(I_{1}+I_{2}+4 I_{3}\right) \alpha^{2}\right)+|\Lambda| \ln (\pi / \alpha)\right\} .
$$

We can see now, that for any $\delta$ there exists $\alpha>0$ which makes the $\beta$-coefficient negative. In other words,

$$
\left\langle P_{\Lambda}\right\rangle_{\Lambda, \beta} \leqq \exp \{-(\beta-C)|\Lambda| x\}, \quad \varkappa, C>0 .
$$

Hence, for $\beta$ sufficiently large the sum (5) is finite and goes to zero as $\beta \rightarrow \infty$, and the proof follows.

\section{Generalizations and Discussions}

3.1. The theorem we have proved is valid in more general setting. Let $G$ be any compact connected Lie group, and $\varphi_{i} \in G$ for any $i \in \mathbb{Z}^{2}$ (instead of $S^{1}$ ). Let $U$ be four-particle interaction:

$$
U_{i}\left(\varphi_{i}, \varphi_{i+e}, \varphi_{i+f}, \varphi_{i+e+f}\right) \in \mathbb{R}^{1}
$$

is the only nonvanishing interaction, $e, f \in \mathbb{Z}^{2}$ are two orthonormal vectors.

We need the following properties of the interaction:

(a) Reflection-invariance:

$$
\begin{aligned}
& U_{i}\left(\varphi_{i}, \varphi_{i+e}, \varphi_{i+f}, \varphi_{i+e+f}\right) \\
& \quad=U_{i+e}\left(\varphi_{i+e}, \varphi_{i}, \varphi_{i+e+f}, \varphi_{i+f}\right) \\
& \quad=U_{i+f}\left(\varphi_{i+f}, \varphi_{i+e+f}, \varphi_{i}, \varphi_{i+e}\right) \text { for any } i .
\end{aligned}
$$

(b) Symmetry:

Let $\mathbb{Z}_{p} \subset G$ be any discrete subgroup of prime order (the existence of them is well-known).

Let us consider the following action of $G \times \mathbb{Z}_{p}$ on the four-spin configuration space $G_{i} \times G_{i+e} \times G_{i+f} \times G_{i+e+f}$ :

$$
\begin{aligned}
& (g, \omega)\left(g_{i}, g_{i+e}, g_{i+f}, g_{i+e+f}\right) \\
& \quad=\left(g^{-1} g_{i}, g^{-1} g_{i+e} \omega, g^{-1} g_{i+f} \omega, g^{-1} g_{i+e+f}\right),
\end{aligned}
$$

where $\omega \in \mathbb{Z}_{p} \subset G$ and $i \in L_{e}$. Let $M$ be the orbit-space of this action, and $e \in M$ be the image of the point $e \times e \times e \times e \in(G)^{4}$ under natural projection map $\pi:(G)^{4} \rightarrow M$. 
We suppose $U_{(0,0)}$ to have a form $\pi^{*} V$, where $V$ is continuous function on $M$ which takes it maximal value exactly in one point $e$.

Then we have the following.

Theorem 2. If the interaction $U$ satisfies conditions 1, 2, then for sufficiently low temperatures we have at least $\varrho$ different Gibbs states for this interaction. If, in addition, the potential is of class $C^{2}$, then all this states are $G$-invariant measures according to natural G-action on the configuration space.

The first statement of the Theorem 2 can be proven exactly in the same way, as the Theorem 1. In fact, Theorem 1 is a corollary of the Theorem 2 (after introducing new coordinates). The second statement of the Theorem 2 follows from the general results of [3].

3.2. The general ideology of two-dimensional systems says that there can be the break down of the hamiltonian symmetry if it is discrete, and it cannot be if it is continuous. So the Theorems 1 and 2 bring a unified example supporting this statement.

3.3. Of course, the statement about absence of breakdown of continuous symmetry is valid only for smooth potentials. It is an interesting open problem to prove, that for nearest-neighbor $S^{1}$-invariant interactions with singularity of the type $\left(-|x|^{\alpha}\right)$, $\alpha<2$ at its maxima, there are infinitely many Gibbs states even in two dimensions. However, there seems to be no methods up to now to attack this problem.

3.4. All the arguments above can be extended to three-dimensional cases. But for this case one has also spontaneous magnetization $\left(G=S^{1}\right)$, as it follows from Ginibre-Dunlop inequalities and infrared bounds. Let $T_{1}$ be the critical temperature for continuous symmetry breaking in three-dimensional version of the model (1), and $T_{2}$ be the critical temperature for discrete symmetry breaking. An interesting open problem is to show that $T_{2}>T_{1}$ (it is easy to see, that $T_{2} \geqq T_{1}$ ).

\section{References}

1. Fröhlich, J., Lieb, E.H.: Commun. Math. Phys. 60, 233-267 (1978)

2. Fröhlich, J., Israel, R., Lieb, E.H., Simon, B.: Commun. Math. Phys. 62, 1-34 (1978)

3. Dobrushin, R.L., Shlosman, S.B.: Commun. Math. Phys. 42, 31-40 (1975)

4. Bricmont, J., Fontaine, J.R., Landau, L.J.: Commun. Math. Phys. 56, 281-296 (1977)

5. Messager, A., Miracle-Sole, S., Pfister, Ch.-E.: Commun. Math. Phys. 58, 19-29 (1978)

6. Pirogov, S.A.: Private communication

7. Georgii, H.-O.: Phasenübergang 1. Art bei Gittergasmodellen. Lecture notes in physics, 16. Eds.: Ehlers, J., Hepp, K., Weidenmüller, H.A. Berlin, Heidelberg, New York: Springer 1972 